\title{
Tale of Two Tails: Molecular Exchange Kinetics of Telechelic Polymer Micelles
}

\author{
Nico König $\odot,{ }^{1,2}$ Lutz Willner $\odot,{ }^{2, *}$ Vitaliy Pipich $\odot,{ }^{3}$ Najet Mahmoudi $\odot,{ }^{4}$ and Reidar Lund $\odot^{1, \dagger}$ \\ ${ }^{1}$ Department of Chemistry, University of Oslo, P.O. Box 1033, Blindern, 0315 Oslo, Norway \\ ${ }^{2}$ Jülich Centre for Neutron Science (JCNS) and Institute for Complex Systems (ICS), \\ Forschungszentrum Jülich GmbH, 52425 Jülich, Germany \\ ${ }^{3}$ Jülich Centre for Neutron Science (JCNS) at Heinz. Maier-Leibnitz Zentrum (MLZ), \\ Forschungszentrum Jülich GmbH, 85747 Garching, Germany \\ ${ }^{4}$ ISIS-STFC, Rutherford Appleton Laboratory, Chilton, Oxon OX11 OQX, United Kingdom
}

(Received 20 December 2019; accepted 14 April 2020; published 15 May 2020)

\begin{abstract}
Telechelic polymers contain two chain ends that are able to promote self-assembly into "flowerlike" or interconnected micellar structures. Here, we investigate the molecular exchange kinetics of such micelles using time-resolved small-angle neutron scattering. We show that the activation energies of monofunctional and telechelic chain exchange are identical. This demonstrates that the two chain ends are not simultaneously released in a single event. Instead, the results show that, contrary to regular micelles, the kinetics occurs in a multistep process involving a collision-induced single-molecule exchange mechanism where the exchange rate is directly proportional to the polymer concentration. We show that this novel mechanism can be quantitatively explained by a simple kinetic model.
\end{abstract}

DOI: 10.1103/PhysRevLett.124.197801

Hydrophobically end-capped polymers, e.g., telechelic polymers bearing two hydrophobic stickers in terminal positions of a water-soluble backbone, are well-studied model systems for self-assembly. Telechelic polymers have attracted a lot of interest because their properties encompass many interesting aspects in soft matter physics, including hierarchical self-assembly, non-Newtonian flow behavior, and colloidal interactions [1-11]. Aside from a purely academic point of view, telechelic polymers are very important as associative thickeners in daily life products, e.g., paints and cosmetics. In water, telechelic polymers spontaneously self-assemble into a variety of different nanostructures. These are, depending on concentration and hydrophobic chain end ("sticker") length, flowerlike micelles, clusters of micelles, and nanostructured hydrogels, where telechelic chains interconnect individual micelles.

All these structures commonly show a continuous exchange of chains to reach and maintain their thermal equilibrium. In recent years, the exchange kinetics of diblock copolymer micelles has been thoroughly studied experimentally and by computer simulation. In particular, time-resolved small-angle neutron scattering (TR-SANS) employing contrast variation proved to be the method of choice to explore the mechanisms of chain exchange under (quasi)equilibrium conditions [12]. TR-SANS applied to different diblock copolymer micellar systems has shown that single-chain expulsion and insertion is the prevailing mechanism for chain exchange with the expulsion as the rate determining step [13-16]-in line with computer simulation $[17,18]$ and scaling theory $[19,20]$. The expulsion is a thermally activated first-order kinetic process, which is characterized by a single exponential relaxation $R(t)=\exp (-k t)$, with $t$ as the time and $k$ as the exchange rate constant. The temperature dependence is expressed in terms of an Arrhenius equation

$$
k=\frac{1}{\tau_{0}} \exp \left(-E_{a} / k_{B} T\right)
$$

with $E_{a}$ as the activation barrier, $\tau_{0}$ as the hypothetical attempt time of the core block to escape from the micelle, and $k_{B} T$ as the thermal energy. $E_{a}$ is essentially determined by the extra interface created by the insoluble block exposed to the solvent. It has been found that $E_{a}$ depends linearly on the length of the insoluble block, which suggests contact between all monomers and solvent rather than collapse into a spherical globule [14,21].

In contrast to amphiphilic diblock copolymers, the exchange mechanism of telechelic micelles in aqueous solution has not yet been determined experimentally. Here, we show experimental evidence of a novel kind of exchange mechanism that consists of a collision-induced multistep process that is fundamentally different from the exchange in regular micelles. Furthermore, we provide a simple kinetic model that describes all experimental data over a range of concentrations and temperatures with a single set of parameters. We believe that the proposed mechanism is relevant to understanding diffusion in other systems where multivalency plays a role, e.g., in supramolecular networks, lipids, and proteins.

In principle, there are three possible scenarios for molecular exchange between telechelic micelles: 
(i) The expulsion of the two core blocks occurs simultaneously. In this case, the exchange rate is expected to be proportional to the product of the two individual rates leading to a process with doubled activation energy compared to diblock exchange.

(ii) The expulsion of the two core blocks proceeds in two consecutive steps with free telechelic chains diffusing in solution. That means the exchange rate exhibits a more complicated temperature dependence and is independent of concentration (see Supplemental Material [22] for a more detailed discussion).

(iii) The expulsion of the two core blocks proceeds in two consecutive steps but with the first block reinserted into another micelle, forming a bridge before the second block escapes. The exchange thus depends on the collision of micelles and the rate would exhibit a clear concentration dependence. Such a mechanism is related to the "walking" diffusion suggested by Yokoyama et al. [23,24] for triblock copolymer melts forming mesocrystal domains.

According to classical transient network theory, the microscopic exchange dynamics in telechelic polymer gels plays a key role for the macroscopic rheological properties [25]. This was recently confirmed by Zinn et al. [10] who found the same $E_{a}$ for the macroscopic relaxation time of a telechelic network determined by linear oscillatory shear experiments and the molecular exchange time for the monofunctional polymers determined by TR-SANS. Lu et al. [26] found that the characteristic exchange time of the triblock copolymer PS-PEP-PS in squalane increases massively compared to a PS-PEP diblock with the same PS block and half the PEP length. The exchange rate was significantly faster than expected for simultaneous release, pointing toward a sequential activation. However, a clear picture for the mechanism is not apparent from that publication.

In this Letter, we present a quantitative description of the exchange kinetics of telechelic polymers in dilute solution based on TR-SANS experiments. They were performed on mixtures of mono- and difunctionalized (telechelic) poly(ethylene oxide) $n$-alkyl ethers: $\mathrm{C}_{n}$-PEO5 and $\mathrm{C}_{n}-\mathrm{PEO} 10-\mathrm{C}_{n}$, with $n=22,28$ the alkane chain lengths and 5 and 10 the approximate PEO molecular weights in $\mathrm{kg} / \mathrm{mol}$. It has been shown by Laflèche et al. [27] that phase separation, usually occurring in aqueous solutions of telechelic polymers, is avoided by blending telechelic and monofunctionalized PEO chains. We utilized this approach in the present study and employed mixtures with volume fractions of the telechelic chains of $f_{\text {tel }}=0.5$ for $\mathrm{C}_{22}$ (C22F50) and $f_{\text {tel }}=0.4$ for $\mathrm{C}_{28}(\mathrm{C} 28 \mathrm{~F} 40)$. Solutions did not show any sign of demixing in a temperature range between 5 and $60^{\circ} \mathrm{C}$, where the TR-SANS experiments have been performed. The structural details of aqueous solutions of the mixtures have been published earlier [28]. In brief, for solutions of $\mathrm{C}_{28}$ mixtures, flowerlike micelles with looped telechelic chains are favored over cluster
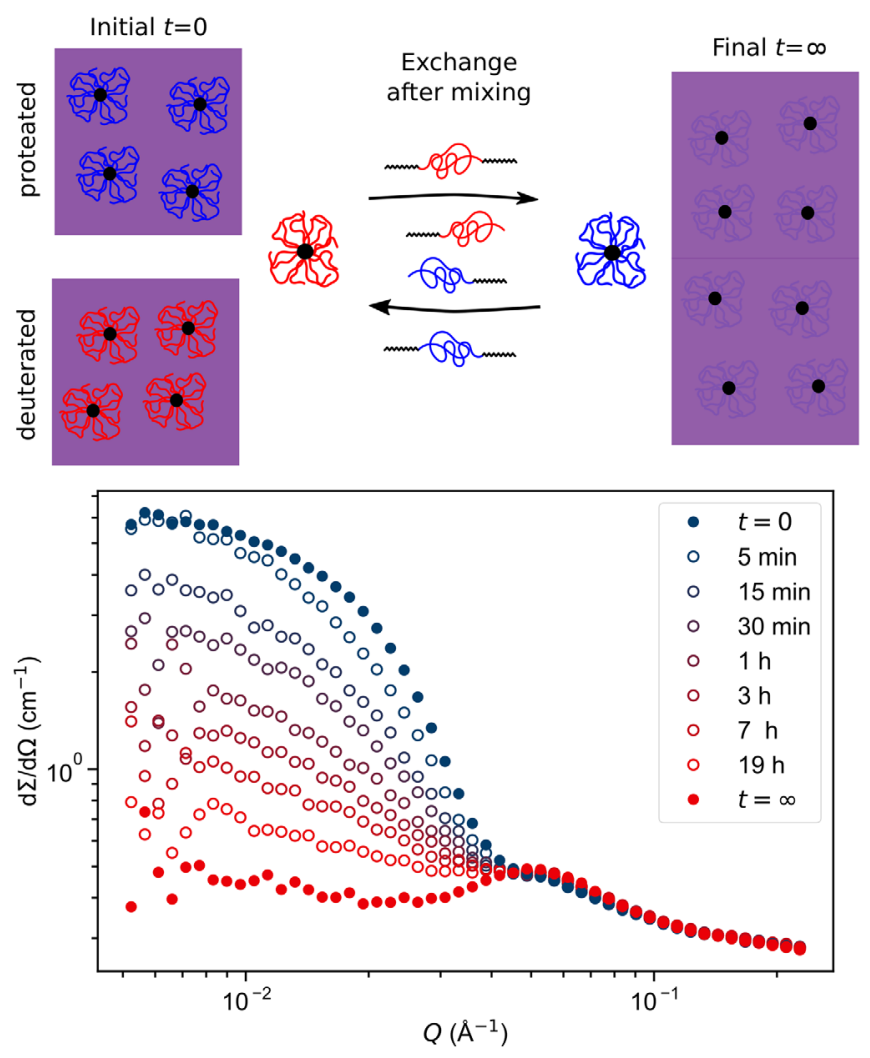

FIG. 1. (Top) Illustration of the kinetic zero average contrast technique to monitor chain exchange in diblock and triblock mixed micelles. (Bottom) Corresponding TR-SANS data of $\mathrm{C}_{28}$-PEO5/ $\mathrm{C}_{28}$-PEO10- $\mathrm{C}_{28}$ micelles obtained at $37^{\circ} \mathrm{C}$ and $1.0 \mathrm{vol} \%$ polymer volume fraction after mixing equal amounts of the deuterated and proteated reservoirs.

formation, which is the principal structure of the $\mathrm{C}_{22}$ samples. An inherent property of this system is partial crystallization of the $n$-alkane chains inside the micellar cores, which leads to a larger activation barrier for exchange amounting to the enthalpy of fusion [21,29]. Accordingly, Eq. (1) needs to be rewritten as

$$
k=\frac{1}{\tau_{0}} \exp \left(\frac{\Delta S_{m}}{k_{B}}\right) \exp \left(-\frac{E_{a}+\Delta H_{m}}{k_{B} T}\right)
$$

with $\Delta H_{m}$ and $\Delta S_{m} \approx \Delta H_{m} / T_{m}$ as the melting enthalpy and entropy, respectively, and $T_{m}$ as the melting temperature.

The exchange of chains was monitored by TR-SANS employing the kinetic zero average contrast (KZAC) technique [30], described in detail in Ref. [12]. The principles of the KZAC technique are schematically illustrated in Fig. 1 (top) for exemplary flowerlike micelles. Two reservoirs, containing proteated and deuterated micelles in an $\mathrm{H}_{2} \mathrm{O} / \mathrm{D}_{2} \mathrm{O}$ solvent mixture with zero contrast to the average of $h$-PEO/d-PEO, are mixed. Initially, the contrast and, correspondingly, the scattered intensity, is maximal, but then decreases as a function of time due to the exchange of mono- and difunctional chains. At $t=\infty$, the 
contrast in the mixture is minimal as the corona chains are matched out by the solvent mixture.

As an example, time-resolved scattering curves are shown in Fig. 1 (bottom) for a 1:1 mixture of $d$ and $h$ $\mathrm{C}_{28}$-PEO5 and $\mathrm{C}_{28}-\mathrm{PEO} 10-\mathrm{C}_{28}$ micelles at $37^{\circ} \mathrm{C}$ and polymer volume fraction $\phi=1.0 \mathrm{vol} \%$. At low scattering vectors $Q$, the plot reveals a continuous decrease of the scattered intensity with time, indicating a decrease of contrast of the PEO corona due to chain exchange. At high $Q$, the intensity is time independent and predominantly results from the proteated $n$-alkane cores and from small contributions of segmental correlations of the PEO polymer [31]. Importantly, the scattered intensity after $19 \mathrm{~h}$ reaches almost the intensity of a preequilibrated sample reflecting the final state. This indicates that both types of chains, monofunctional and telechelic, are exchanging within the experimentally accessible time window.

To simplify data analysis, only the integrated scattering intensity

$$
I(t)=\int \frac{d \Sigma}{d \Omega}(Q, t) d Q
$$

was used, with $d \Sigma / d \Omega(Q, t)$ as the time-dependent macroscopic scattering cross section, i.e., the scattering intensity. This procedure is equivalent to analyzing the full scattering curve [15]. The integrated intensities were then analyzed in terms of a dimensionless relaxation function $R(t)$ defined as

$$
R(t)=\sqrt{\frac{I(t)-I(\infty)}{I(0)-I(\infty)},}
$$

where $I(0)$ denotes the intensity at $t=0$ and $I(\infty)$ is the intensity of a preequilibrated sample. Figure 2 depicts typical relaxation curves of $\mathrm{C} 22 \mathrm{~F} 50$ at different temperatures.

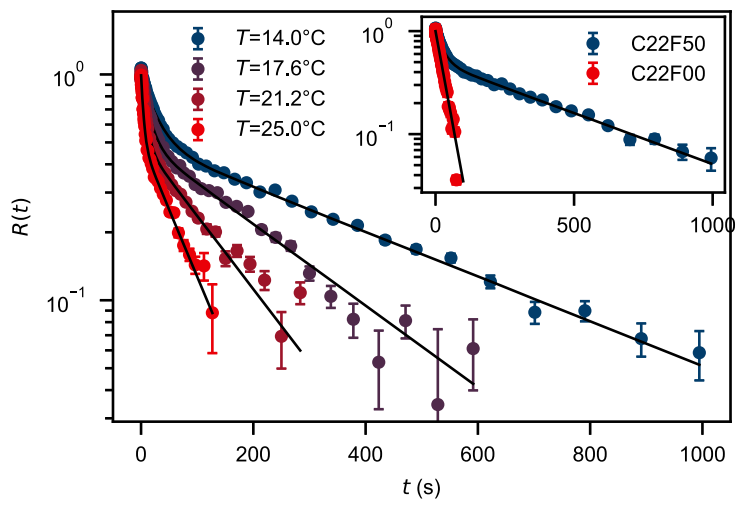

FIG. 2. Relaxation functions $R(t)$ of $\mathrm{C} 22 \mathrm{~F} 50$ at selected temperatures. Black lines represent fits according to Eq. (5). (Inset) Comparison of $R(t)$ of $\mathrm{C} 22 \mathrm{~F} 50$ and $\mathrm{C} 22 \mathrm{~F} 00$ (pure monofunctional) at $14{ }^{\circ} \mathrm{C}$. The data were obtained using a stopped-flow mixing device.
The plot clearly reveals two well-separated processes, which we intuitively assign to the exchange of monofunctional $\mathrm{C}_{22}-\mathrm{PEO} 5$ and difunctional $\mathrm{C}_{22}-\mathrm{PEO} 10-\mathrm{C}_{22}$ copolymers, respectively. The assignment is further supported by comparing $R(t)$ of $\mathrm{C} 22 \mathrm{~F} 50$ with that of pure $\mathrm{C} 22-\mathrm{PEO} 5$ micelles (C22F00) at $T=14^{\circ} \mathrm{C}$, as depicted in the inset of Fig. 2. The plot shows that $R(t)$ of $\mathrm{C} 22 \mathrm{~F} 00$ coincides with the fast process of the mixture. Based on this, we have fitted the relaxation curves by a sum of two exponential functions, with prefactors corresponding to the volume fraction $f_{\text {tel }}$ of the mixture

$$
R(t)=f_{\text {tel }} \exp \left(-k_{\text {tel }} t\right)+\left(1-f_{\text {tel }}\right) \cdot \exp \left(-k_{\text {mon }} t\right) .
$$

Excellent fits were obtained as shown with solid lines in Fig. 2, yielding the rate constants for monofunctional $k_{\text {mon }}$ and telechelic $k_{\text {tel }}$ chain exchange at each temperature. A preliminary Arrhenius evaluation of the fitted rate constants has shown that the $E_{a}$ for chain escape is identical for both mono- and difunctional polymers. Subsequently, the data could be reanalyzed to further stabilize the fit using $E_{a}=$ $E_{a \text {,mon }}=E_{a \text {,el }}$ and perform simultaneous fits at all temperatures. Thus, only $E_{a}, \tau_{0, \text { mon }}$, and $\tau_{0 \text {,tel }}$ were free fit parameters. $T_{m}$ and $\Delta H_{m}$ have been determined independently by calorimetry as described recently [31]; the data are shown in Fig. S3 in the Supplemental Material [22], together with experimental relaxation curves and fits for each individual temperature for both polymers (Figs. S1 and S2). Fit values and thermodynamic parameters are summarized in Table I.

Arrhenius plots of the fitted parameters of mono- and difunctional chain exchange are presented for $\mathrm{C}_{22}$ and $\mathrm{C}_{28}$ in Fig. 3, together with rate constants obtained from an earlier study [29] of pure $\mathrm{C}_{n}$-PEO5 polymers for comparison. The Arrhenius representations display two parallel lines, as $E_{a}$ was found to be identical for monofunctional and telechelic chain exchange. The numerical values of $E_{a}$

TABLE I. Parameters of simultaneous fits at $\phi=1 \mathrm{vol} \%$ and different temperatures as well as important thermodynamic parameters of $\mathrm{C}_{28}$ and $\mathrm{C}_{22}$ polymer mixtures. Values for monofunctional polymer micelles are taken from a previous study [29] and added for comparison.

\begin{tabular}{lcccc}
\hline \hline & $\mathrm{C} 22 \mathrm{~F} 50$ & $\mathrm{C} 22 \mathrm{~F} 00^{\mathrm{a}}$ & $\mathrm{C} 28 \mathrm{~F} 40$ & ${\mathrm{C} 28 \mathrm{~F} 00^{\mathrm{a}}}^{\mathrm{a}}$ \\
\hline$-\log _{10}\left[\tau_{0, \text { mon }}(\mathrm{s})\right]$ & $15.5 \pm 1.2$ & $13.5 \pm 3.0$ & $18.7 \pm 1.5$ & $19.7 \pm 2.5$ \\
$\left.-\log _{10}\left[\tau_{0, \text { tel }} \mathrm{s}\right)\right]$ & $14.3 \pm 1.3$ & & $17.1 \pm 1.6$ & \\
$E_{a}(\mathrm{~kJ} / \mathrm{mol})$ & $92 \pm 7$ & $80 \pm 15$ & $125 \pm 10$ & $130 \pm 15$ \\
$T_{m}\left({ }^{\circ} \mathrm{C}\right)$ & $29 \pm 1$ & $29 \pm 1$ & $57 \pm 1$ & $57 \pm 1$ \\
$\Delta H_{m}(\mathrm{~kJ} / \mathrm{mol})$ & $24 \pm 1$ & $25 \pm 1$ & $40 \pm 3$ & $40 \pm 3$ \\
$\Delta S_{m}(\mathrm{~J} / \mathrm{mol} / \mathrm{K})$ & $79 \pm 2$ & $84 \pm 2$ & $121 \pm 8$ & $121 \pm 8$ \\
$\tau_{1 / 2 \text {,mon }}(\mathrm{s})^{\mathrm{b}}$ & 4.6 & 5.1 & $7.9 \times 10^{3}$ & $6.3 \times 10^{3}$ \\
$\tau_{1 / 2, \text { tel }}(\mathrm{s})^{\mathrm{b}}$ & 73 & & $3.0 \times 10^{5}$ & \\
\hline \hline
\end{tabular}

Taken from Ref. [29].

${ }^{\mathrm{b}}$ Half-exchange time calculated for $T=25^{\circ} \mathrm{C}$ via Eq. (2). 

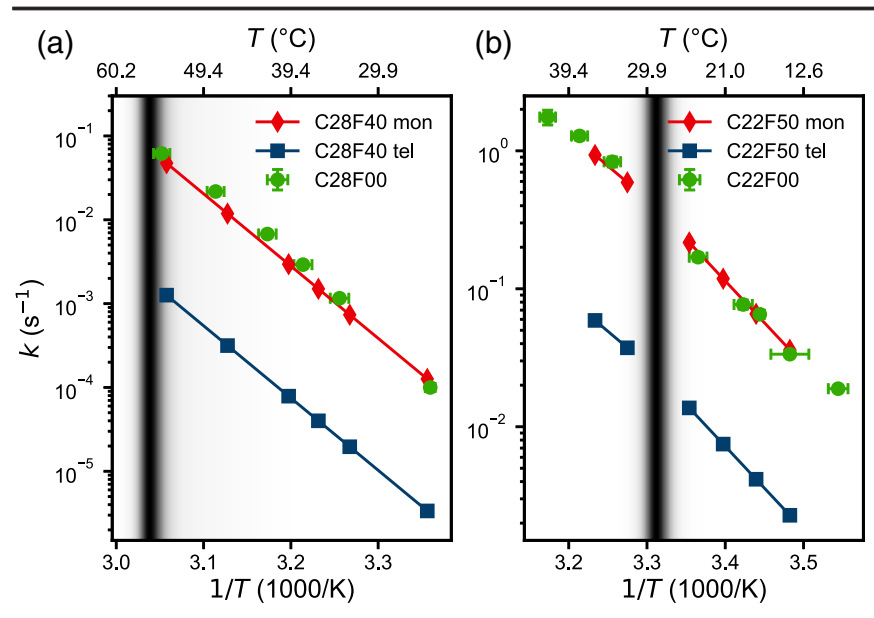

FIG. 3. Arrhenius representation of the rate constants of $\mathrm{C}_{28}$ and $\mathrm{C}_{22}$ monofunctional (mon) and telechelic (tel) polymers, obtained via a simultaneous fit of all temperatures. For comparison, previous data of the pure monofunctional samples (F00) are included [29]. The gray shades are contour plots of differential scanning calorimetry traces, indicating the melting transitions of the micellar cores.

are in good agreement with values obtained earlier for pure diblock polymer exchange [21,29], see Table I. Because of the exponential dependence, the obtained attempt times $\tau_{0}$ are rather uncertain, but still similar to previous results. Following the assumptions made above, we can unambiguously rule out the simultaneous escape of the two core blocks, as this would correspond to twice the activation energy.

From the above results, we can already conclude that the exchange of telechelic chains proceeds via a consecutive release of the two chain ends. Either the two hydrophobic blocks are released stepwise and then diffuse freely [scenario (ii)] or short-lived clusters are formed [scenario (iii)]. Now, in order to distinguish between the two mechanisms, we have measured $\mathrm{C}_{28}$ relaxation curves at different concentrations because the latter mechanism is collision based and therefore concentration dependent. Figure 4 shows relaxation curves of $\mathrm{C}_{28}-\mathrm{PEO} 5$ and $\mathrm{C}_{28}$-PEO10- $\mathrm{C}_{28}$ micelles measured at three different polymer volume fractions, $\phi=0.5,1.0$, and $2.5 \mathrm{vol} \%$.

As seen, the three relaxation curves superimpose at short times, indicating a concentration-independent escape of monofunctional PEO chains, whereas the telechelic chains show a distinct acceleration of chain exchange with increasing $\phi$. The kinetic data were quantitatively analyzed by fitting with Eq. (5) and the obtained rate constants extracted for the fast and slow process are plotted vs $\phi$ in the inset of Fig. 4. The rate constant of the fast process, associated with $k_{\text {mon }}$, is basically constant, which confirms that the exchange is not affected by micellar collisions. However, the slow process, associated with $k_{\text {tel }}$, on the other hand, scales linearly with $\phi$, suggesting that collisioninduced chain exchange is the dominating mechanism for telechelic chain exchange. To further confirm the proposed

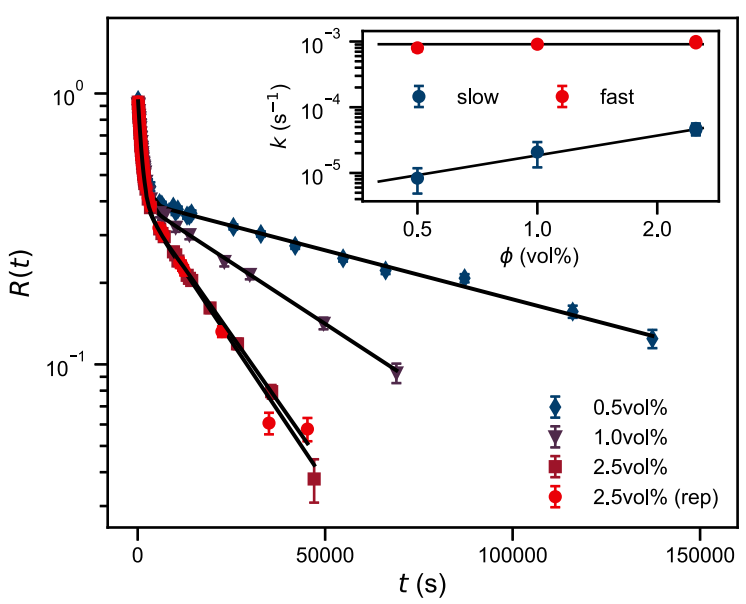

FIG. 4. Relaxation curves of $\mathrm{C} 28 \mathrm{~F} 40$ measured at $T=37^{\circ} \mathrm{C}$ and three different polymer volume fractions. (Inset) Respective rate constants obtained by fitting Eq. (5). The fast process is concentration independent, while the slow process exhibits a $k \sim \phi$ dependence.

exchange mechanism, we performed concentration- and temperature-dependent measurements on C28F40compare Figs. S4 and S5 in the Supplemental Material [22]. We were able to fit all relaxation curves with one set of parameters, in agreement with the values given in Table I. As expected, $E_{a}$ is constant for all three concentrations, proving that the collision-induced exchange is the dominant exchange mechanism for telechelic micelles.

The proposed mechanism is similar to the walking diffusion suggested by Yokoyama et al. $[23,24]$ in polymer melts, but differs by the lack of micellar crystalline order. Instead, the exchange events are triggered by random collisions between micelles where at least one chain is in an "activated state," i.e., with one chain end outside the micelle. It also resembles collision-based exchange mechanisms recently found in lipid nanodisks [32]. For the present case of telechelic molecules in solution, we propose the three-step kinetic model sketched in Fig. 5. This can be written as (1) expulsion of one telechelic hydrophobic

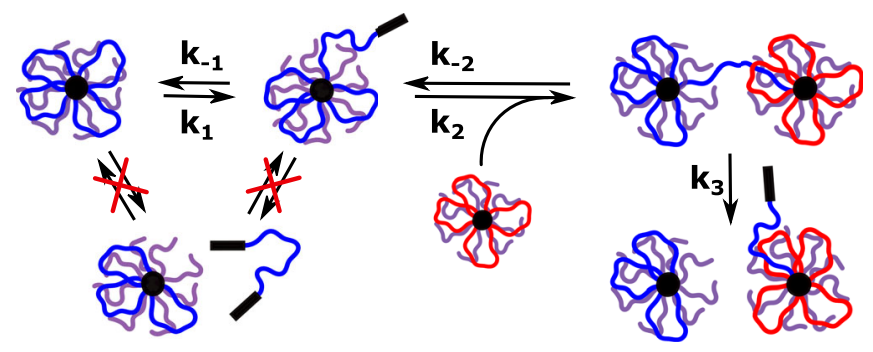

FIG. 5. Visualization of the exchange mechanism of telechelic chains in flowerlike micelles and clusters of micelles via a sequence of consecutive equilibrium steps involving collisioninduced exchange. Diffusion of free telechelic chains as an important pathway is excluded. 
chain end while the other one is still buried in the micellar core,

$$
P \underset{k_{-1}}{\stackrel{k_{1}}{\rightleftharpoons}} P^{*}
$$

(2) insertion of the free chain end into another micelle, i.e., bridging

$$
P^{*}+M \underset{k_{-2}}{\stackrel{k_{2}}{\rightleftharpoons}} P^{* *},
$$

and (3) release of the second chain end that was still in the original micelle,

$$
P^{* *} \stackrel{k_{3}}{\rightarrow} M+\bar{P}^{*}
$$

Here, $P$ represents telechelic polymer molecules that have not exchanged yet and both chain ends are buried in the original micelle. $P^{*}$ are molecules that are also still attached to their original micelle but with one chain end free in solution and $M$ is an arbitrary micelle. $P^{* *}$ is a molecule that bridges between its original micelle and another one and $\bar{P}^{*}$ is a chain that has exchanged at least once and has one free chain end.

Applying a steady-state approximation for the unstable intermediates $P^{*}$ and $P^{* *}$, this leads to a single exponential (see Supplemental Material [22] for a more detailed discussion)

$$
[P](t)=[P]_{0} \exp \left(-k_{\mathrm{eff}} t\right)
$$

with the concentration-dependent decay constant $k_{\text {eff }}=k_{1} k_{2}[M] /\left(2 k_{-1}+k_{2}[M]\right) .[M]=\phi /\left(N_{\text {agg }} N_{\text {avo }} V_{p}\right)$ where $N_{a g g}$ is the aggregation number, $V_{p}$ is the volume of a chain and $N_{a v o}$ is Avagadros number. For low micelle concentrations and $k_{2} \leq k_{-1}$, a Taylor expansion in [M] yields $k_{\text {eff }}=k_{1} k_{2}[M] /\left(2 k_{-1}\right)+\mathcal{O}\left([M]^{2}\right)$. Thus, in the leading order, the rate constant depends linearly on $[M]$, concurring with our experimental data shown in the inset of Fig. 4, as well as Figs. S4 and S5 in the Supplemental Material [22]. Since $k_{-1}$ and $k_{2}$ have approximately the same enthalpic contribution, the temperature dependence is governed by $k_{1}$. Moreover, the model provides an equilibrium constant $K=k_{1} / k_{-1} \ll 1$, which controls release of a single chain end. This naturally describes the effective reduction of the exchange rate of telechelic chains, $k_{\text {eff }}=K k_{2}[M] / 2$. These findings are in perfect agreement with our experimental results. In addition, the telechelic PEO block is longer than the corresponding monofunctional one, which is known to slow down chain exchange [19,33].

In conclusion, we have shown that the molecular exchange of telechelic polymers in flowerlike micelles and clusters of micelles proceeds in consecutive steps via a collision-induced single-chain exchange mechanism that so far has not been reported experimentally. TR-SANS in combination with the KZAC contrast variation method was applied to monitor the chain exchange in a well-defined model system for self-assembly. By carefully analyzing the TR-SANS data at different temperatures and concentrations, we can rule out a simultaneous release of the two chain ends. In addition, we have developed a kinetic model that explains the experimental findings and provides a rather complete picture of the equilibration mechanism and diffusion processes in such systems. The discovered collision-induced exchange mechanism is relevant also to other self-assembled structures, in which multivalency plays a role, e.g., in supramolecular networks, lipid membranes, multidomain proteins, lipoproteins, and conventional hydrogels.

This work is based on experiments performed at the KWS-2 instrument [34] operated by JCNS at Heinz MaierLeibnitz Zentrum, Garching, Germany, and the Sans2d instrument [35,36] at STFC ISIS Neutron and Muon Source, United Kingdom. We thank Bente A. Breiby (School of Pharmacy, UiO) and Thomas Zinn (ESRFThe European Synchrotron) for providing the differential scanning calorimetry data.

"1.willner@fz-juelich.de reidar.lund@kjemi.uio.no

[1] T. Annable, R. Buscall, R. Ettelaie, and D. Whittlestone, The rheology of solutions of associating polymers: Comparison of experimental behavior with transient network theory, J. Rheol. 37, 695 (1993).

[2] A. N. Semenov, J.-F. Joanny, and A. R. Khokhlov, Associating polymers: Equilibrium and linear viscoelasticity, Macromolecules 28, 1066 (1995).

[3] E. Alami, M. Almgren, W. Brown, and J. François, Aggregation of hydrophobically end-capped poly(ethylene oxide) in aqueous solutions. fluorescence and light-scattering studies, Macromolecules 29, 2229 (1996).

[4] E. Beaudoin, O. Borisov, A. Lapp, L. Billon, R. C. Hiorns, and J. François, Neutron scattering of hydrophobically modified poly(ethylene oxide) in aqueous solutions, Macromolecules 35, 7436 (2002).

[5] J. Berret, Fluorocarbon associative polymers, Curr. Opin. Colloid Interface Sci. 8, 296 (2003).

[6] X.-X. Meng and W. B. Russel, Rheology of telechelic associative polymers in aqueous solutions, J. Rheol. 50, 189 (2006).

[7] T. Koga, F. Tanaka, R. Motokawa, S. Koizumi, and F. M. Winnik, Theoretical modeling of associated structures in aqueous solutions of hydrophobically modified telechelic PNIPAM based on a neutron scattering study, Macromolecules 41, 9413 (2008).

[8] J. Sprakel, E. Bartscherer, G. Hoffmann, M. A. C. Stuart, and J. van der Gucht, Dynamics of polymer bridge formation and disruption, Phys. Rev. E 78, 040802 (2008). 
[9] J. Sprakel, E. Spruijt, M. A. C. Stuart, N. A. M. Besseling, M. P. Lettinga, and J. van der Gucht, Shear banding and rheochaos in associative polymer networks, Soft Matter 4, 1696 (2008).

[10] T. Zinn, L. Willner, and R. Lund, Telechelic polymer hydrogels: Relation between the microscopic dynamics and macroscopic viscoelastic response, ACS Macro Lett. 5, 1353 (2016).

[11] G. W. Park and G. Ianniruberto, A new stochastic simulation for the rheology of telechelic associating polymers, J. Rheol. 61, 1293 (2017).

[12] R. Lund, L. Willner, and D. Richter, Kinetics of block copolymer micelles studied by small-angle scattering methods, Adv. Polym. Sci. 259, 51 (2013).

[13] R. Lund, L. Willner, D. Richter, and E. E. Dormidontova, Equilibrium chain exchange kinetics of diblock copolymer micelles: Tuning and logarithmic relaxation, Macromolecules 39, 4566 (2006).

[14] S.-H. Choi, T. P. Lodge, and F. S. Bates, Mechanism of Molecular Exchange in Diblock Copolymer Micelles: Hypersensitivity to Core Chain Length, Phys. Rev. Lett. 104, 047802 (2010).

[15] T. Zinn, L. Willner, R. Lund, V. Pipich, and D. Richter, Equilibrium exchange kinetics in n-alkyl-PEO polymeric micelles: Single exponential relaxation and chain length dependence, Soft Matter 8, 623 (2012).

[16] J. Lu, F. S. Bates, and T. P. Lodge, Chain exchange in binary copolymer micelles at equilibrium: Confirmation of the independent chain hypothesis, ACS Macro Lett. 2, 451 (2013).

[17] Z. Li and E. E. Dormidontova, Equilibrium chain exchange kinetics in block copolymer micelle solutions by dissipative particle dynamics simulations, Soft Matter 7, 4179 (2011).

[18] A. Prhashanna, S. A. Khan, and S. B. Chen, Kinetics of chain exchange between diblock copolymer micelles, Macromol. Theory Simul. 25, 383 (2016).

[19] A. Halperin and S. Alexander, Polymeric micelles: Their relaxation kinetics, Macromolecules 22, 2403 (1989).

[20] A. Halperin, On micellar exchange: The role of the insertion penalty, Macromolecules 44, 5072 (2011).

[21] T. Zinn, L. Willner, V. Pipich, D. Richter, and R. Lund, Effect of core crystallization and conformational entropy on the molecular exchange kinetics of polymeric micelles, ACS Macro Lett. 4, 651 (2015).

[22] See Supplemental Material at http://link.aps.org/ supplemental/10.1103/PhysRevLett.124.197801 for additional TR-SANS data, differential scanning calorimetry curves as well as a detailed discussion of exchange scenarios (ii) and (iii).

[23] H. Yokoyama and E. J. Kramer, Diffusion of triblock copolymers in a spherical domain structure, Macromolecules 33, 954 (2000).
[24] H. Yokoyama, E. J. Kramer, and G. H. Fredrickson, Simulation of diffusion of asymmetric diblock and triblock copolymers in a spherical domain structure, Macromolecules 33, 2249 (2000).

[25] F. Tanaka and S. F. Edwards, Viscoelastic properties of physically crosslinked networks. 1. Transient network theory, Macromolecules 25, 1516 (1992).

[26] J. Lu, F. S. Bates, and T. P. Lodge, Remarkable effect of molecular architecture on chain exchange in triblock copolymer micelles, Macromolecules 48, 2667 (2015).

[27] F. Laflèche, D. Durand, and T. Nicolai, Association of adhesive spheres formed by hydrophobically end-capped PEO. 1. Influence of the presence of single end-capped PEO, Macromolecules 36, 1331 (2003).

[28] T. Zinn, L. Willner, K. D. Knudsen, and R. Lund, Selfassembly of mixtures of telechelic and monofunctional amphiphilic polymers in water: From clusters to flowerlike micelles, Macromolecules 50, 7321 (2017).

[29] N. König, L. Willner, V. Pipich, T. Zinn, and R. Lund, Cooperativity During Melting and Molecular Exchange in Micelles with Crystalline Cores, Phys. Rev. Lett. 122, 078001 (2019).

[30] L. Willner, A. Poppe, J. Allgaier, M. Monkenbusch, and D. Richter, Time-resolved SANS for the determination of unimer exchange kinetics in block copolymer micelles, Europhys. Lett. 55, 667 (2001).

[31] König, L. Willner, and R. Lund, Structure and thermodynamics of mixed polymeric micelles with crystalline cores: Tuning properties via co-assembly, Soft Matter 15, 7777 (2019).

[32] R. Cuevas Arenas, B. Danielczak, A. Martel, L. Porcar, C. Breyton, C. Ebel, and S. Keller, Fast collisional lipid transfer among polymer-bounded nanodiscs, Sci. Rep. 7, 45875 (2017).

[33] T. Zinn, L. Willner, V. Pipich, D. Richter, and R. Lund, Molecular exchange kinetics of micelles: Corona chain length dependence, ACS Macro Lett. 5, 884 (2016).

[34] A. Radulescu, N. K. Szekely, and M.-S. Appavou, KWS-2: Small angle scattering diffractometer, J. Large-Scale Res. Facil. 1, A29 (2015).

[35] R. Lund, N. König, N. Mahmoudi, L. Willner, and L. Willner, Tuning the Molecular Exchange Kinetics of SelfAssembled Antimicrobial Peptide-Polymer Conjugates (STFC ISIS Neutron and Muon Source, 2019), https://doi .org/10.5286/ISIS.E.RB1910106.

[36] R. Lund, N. König, L. Cavalcanti, L. Willner, H. Sørensen, and L. Willner, Exchange Kinetics of Telechelic Cn-PEOCn Micelles: Effect of Conformation and Crystallinity (STFC ISIS Neutron and Muon Source, 2019), https://doi .org/10.5286/ISIS.E.RB1920656. 